\title{
Mechanical Property Evolution of Polymeric Composites Immersed in Jet Fuel
}

\author{
M.R. Condruz ${ }^{a, *}$, S.I. Vintilă ${ }^{a}$, A. Paraschiv ${ }^{a}$, C. PuşCaşu ${ }^{a}$ And F. Dumitru ${ }^{b}$ \\ ${ }^{a}$ Romanian Research \& Development Institute for Gas Turbines - COMOTI, Bucharest, Romania \\ ${ }^{b} \mathrm{SC}$ Autonomous Flight Technologies R\&D SRL, Bucharest, Romania
}

\begin{abstract}
The aim of the present study is to evaluate the jet fuel influence on tensile strength of a polymeric composite material. To accomplish the goal, composite samples were immersed in a kerosene-type jet fuel (JET A-1) for a period of 137 days at room temperature. Three sample batches were studied: references, immersed samples and immersed samples which were vacuum dried. Tensile tests were carried out on each sample batch, and it was concluded that the jet fuel reduces the tensile strength of the epoxy composite while increasing the elastic modulus. The accelerated dry process ensures the partial desorption of kerosene, but it does not ensure an improvement of composite mechanical properties. The overall conclusion of the study is that jet fuel has a negative influence over the studied bare carbon fiber reinforced polymer tensile strength on long term due to degradation of polymeric matrix.
\end{abstract}

DOI: 10.12693/APhysPolA.135.965

PACS/topics: jet fuel, composites, tensile strength, moisture, polymers

\section{Introduction}

All industrial sectors are continuously developing and as a result, the demand for new materials with better properties is increasing. The new materials should ensure high strength, environmental resistance, low density that result in a decrease of weight, reduction of energy/fuel consumption and reduction of maintenance costs. One of the main material categories that are currently studied to replace conventional materials are polymeric composites, especially carbon-fiber reinforced polymers (CFRP). Composites are currently used to manufacture tank shells used for compressed hydrogen storage [1] and they can be studied to be integrated in jet fuel tank assemblies. For this application, an environmental compatibility evaluation is required due to moisture impact on material properties. Environmental degradation is a significant phenomenon that affects polymeric composites, especially the polymeric matrix. Moisture uptake in organic polymers leads to plasticization or hydrolysis which can cause material failure caused by significant reduction of mechanical properties and glass transition temperature $\left(T_{g}\right)$ [2]. Researches were conducted in the last two decades regarding moisture absorption influence on mechanical properties of polymeric composites [38], all resulting in degradation of material's mechanical properties. The main factors that influenced the degradation were: time of exposure (properties are reduced after short-term exposure while damage becomes more significant after long term exposure), temperature (a higher temperature increase properties degradation rate), sample geometry, void content and reinforcement orientation.

*corresponding author; e-mail: raluca. condruz@comoti.ro
The goal of the present research was to evaluate the impact of jet fuel on tensile strength of a polymeric composite reinforced with carbon fibers and to analyze if the effect of fuel is reversible.

\section{Experimental procedure}

For this study, a carbon fiber reinforced epoxy composite material (HexPly M79/42\%/200T2X2/CHS-3K prepreg) was used to manufacture composite laminates. Eight prepreg plies were laid-up at $0^{\circ}$ on metallic moulds and cured in a Scholz autoclave (curing cycle: heating up from room temperature with a heating rate of $3{ }^{\circ} \mathrm{C} / \mathrm{min}$ until reaching $90^{\circ} \mathrm{C}$, holding $4 \mathrm{~h}$ at a pressure of 3 bar and -0.9 bar vacuum level, cooling down until room temperature with a cooling rate of $3^{\circ} \mathrm{C} / \mathrm{min}$ ). Standard tensile test specimens, based on ISO: 527-4: 2000 and ASTM D3039/D3039M: 2000, were cut from laminates using a water jet cutting machine. To eliminate residual water contained by the specimens, they were postcured in a vacuum assisted oven (curing cycle: heating up from room temperature until $120^{\circ} \mathrm{C}$, holding for $2 \mathrm{~h}$, and afterwards oven assisted cooled). Jet fuel effect on material's tensile strength was evaluated by full specimen immersion in kerosene-type jet fuel (JET A-1). Two sample batches were immersed simultaneous and hold in kerosene at room temperature $\left(25 \pm 5^{\circ} \mathrm{C}\right)$ for $3288 \mathrm{~h}$ in an enclosed container and one sample batch was kept unimmersed as reference. The samples were removed from the kerosene container and wiped with a cloth. One sample batch was further vacuum bagged and introduced in an oven at $65^{\circ} \mathrm{C}$ for one hour. All the samples were weighted out before and after immersion and also after the accelerated vacuum drying process to determine the moisture uptake of the material. A visual inspection and a dimensional analysis by caliper measurement were performed before and after the samples were immersed in jet fuel. 
Tensile tests were carried out on each sample batch using a universal mechanical testing machine Instron 8802 (250 kN load cell) with a displacement rate of $2 \mathrm{~mm} / \mathrm{min}$.

\section{Results and discussion}

The visual inspection of the composite samples after they were removed from the moist environment showed no surface damage, the material was not swollen, moreover cracks or peelings were not observed. The kerosene from the container was clear, pieces/particles from the specimens were not observed. This observation proves that noticeable physical damage of the material wasn't encountered. Specimen dimensional analysis sustained the visual inspection results. Regarding the moisture uptake analysis, a $0.44 \mathrm{wt} \%$ moisture uptake was observed after the samples were removed from the container. In case of vacuum dried samples, only a $0.14 \mathrm{wt} \%$ moisture uptake was encountered which means that accelerated dry process ensures the partial desorption of kerosene.

Tensile tests showed that majority of the samples yield in multiple areas, especially near or inside grip/tab area, or near the middle of the sample. Mechanical characterization showed a slight reduction of tensile strength after kerosene immersion. In case of the references a medium tensile strength of $905 \mathrm{MPa}$ and an elastic modulus of $70 \mathrm{GPa}$ were obtained, while in case of the kerosene immersed samples a medium tensile strength of $845 \mathrm{MPa}$ and an elastic modulus of $74 \mathrm{GPa}$ were obtained.

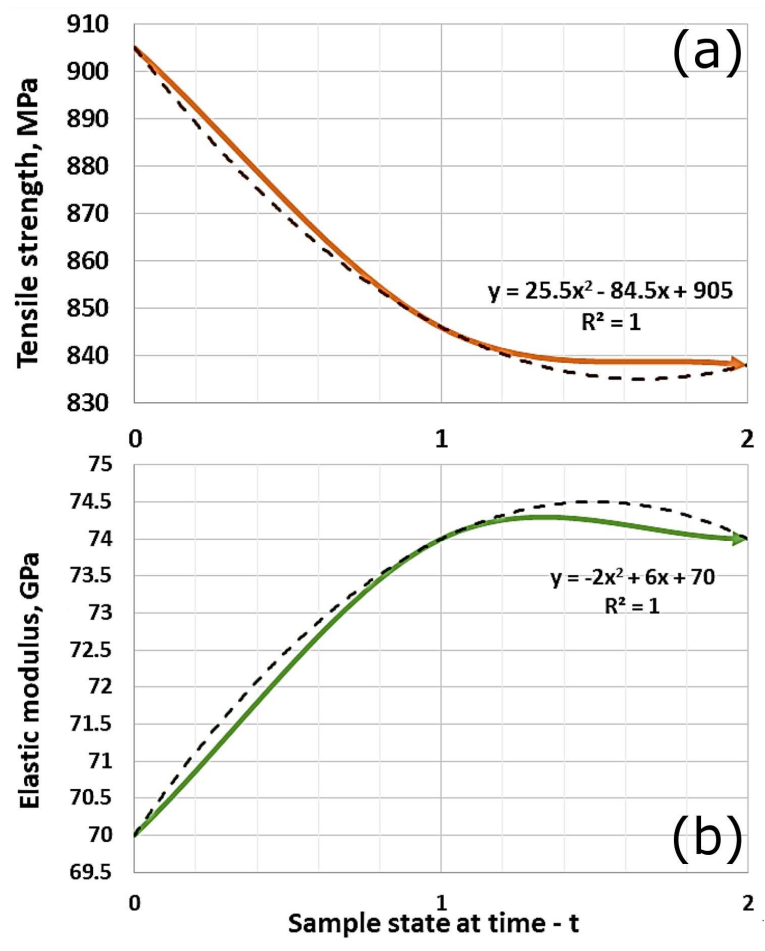

Fig. 1. (a) Influence of kerosene on composite tensile strength; (b) Influence of kerosene on composite elastic modulus (sample state at time $t$ : 0 - unimmersed, 1 - immersed, 2 - vacuum dried).
In case of kerosene immersed and vacuum dried samples, a medium tensile strength of $838 \mathrm{MPa}$ and an elastic modulus of $74 \mathrm{MPa}$ were observed, the modulus values were kept after the vacuum drying process, but the mechanical strength continues to degrade. Even if the heat ensures desorption of the kerosene contained by the matrix, temperature amplifies the negative influence of the fuel on CFRP properties. The mechanical characterization results are presented in Fig. 1a, b. The experimental results obtained in this study are in accordance with the results found in the literature $[8,9]$. The decrease of tensile strength and increase of elastic modulus are caused by the moisture absorbed in the epoxy matrix during the immersion in kerosene. The moisture was absorbed by the polymeric matrix and it infiltrated into the material pores. The matrix properties were reduced, this causing the reduction of the overall mechanical strength of the composite. It was observed that in case of HexPly M79, reduction of mechanical properties is progressive and influenced by temperature, but further studies should be made to determine the behavior of this material in another environmental condition (different environment, temperature range) or in jet fuel for a longer period of time to determine if it is suitable for tanks.

\section{Conclusion}

In this study was concluded that the effect of JET A-1 on polymeric composite is deleterious, even if the physical changes (moisture content) can be reversible, the effect of jet fuel on mechanical properties is permanent. Moisture absorption analysis showed a $0.44 \mathrm{wt} \%$ moisture content after $3288 \mathrm{~h}$ immersion in kerosene. Temperature and vacuum exposure after immersion ensures an accelerated dry process which result in a reduction of moisture content. Mechanical characterization showed an increase with $6 \%$ of elastic modulus values after kerosene immersion with and without temperature exposure, a decrease of $6 \%$ of tensile strength was noticed in case of immersed samples while a decrease of $7 \%$ of tensile strength was noticed in case of immersed and vacuum dried samples. As a general conclusion kerosene has a negative influence over the studied bare CFRP tensile strength on long term due to the degradation of polymeric matrix by infiltration into the material internal pores. Jet fuel degrades the mechanical strength and cause plasticization of the polymer. Even if the heat ensures an evaporation of the kerosene contained by the matrix, temperature amplifies the negative influence of the fuel on CFRP properties. Consequently, surface protection of CFRP should be considered in order to avoid the direct contact with jet fuels.

\section{Acknowledgments}

This work was carried out within POC-A1-A1.2.3G-2015, ID/SMIS code: P_40_422/105884, "TRANSCUMAT" Project, Grant no. $114 / 09.09 .2016$ (Subsidiary Contract no. 2/D.1.6/114/24.10.2017), Project supported by the Romanian Minister of Research and Innovation. 


\section{References}

[1] Report of the Basic Energy Science Workshop for Materials under Extreme Environments, Office of Basic Energy Sciences, U.S. DOE, Washington 2008.

[2] S. Roy, Moisture-Induced Degradation, in: Longterm Durability of Polymeric Matrix Composites, Eds. K.V. Pochiraju, G.P. Tandon, G.A. Schoeppner, Springer US, Boston (MA) 2012, p. 181.

[3] M. Meng, M.J. Rizvi, S.M. Grove, H.R. Le, Comp. Struct. 133, 1024 (2015).

[4] M.L. Costa, M.C. Rezende, S. Almeida, Polym.-Plast. Technol. Eng. 45, 691 (2006).
[5] M.L. Costa, S.F.M. Almeida, M.C. Rezende, Mat. Res. 8, 335 (2005).

[6] J.M Ryan, R. Adams, S.G. Brown, Moisture Ingress Effect on Properties of CFRP, International Committee on Composite Materials, UK 2009.

[7] S.A. Grammatikos, B. Zafari, M.C. Evernden, J.T. Mottram, J.M. Mitchels, Polym. Degrad. Stabil. 121, 407 (2015).

[8] A. Pandian, M. Vairavan, W.J.J. Thangaiah, M. Uthayakumar, J. Comp. 2014, 587980 (2014).

[9] E.P. Otaluka, C. Arnold, S. Alston, in: Proc. 10th Int. Conf. Comp. Sci. Techn., ICCST/10, Lisbon 2015, paper 208. 